\title{
VALIDAÇÃO DO DIAGNÓSTICO DOR ÓSSEA E SUAS INTERVENÇÕES DE ENFERMAGEM NO MIELOMA MÚLTIPLO*
}

Luiz Fernandes Gonçalves Fialho', Patricia dos Santos Claro Fuly², Mauro Leonardo Salvador Caldeira dos Santos³, Joséte Luzia Leite ${ }^{4}$, Silvia Maria de Sá Basílio Lins ${ }^{5}$

${ }^{1}$ Enfermeiro. Mestre em enfermagem. Universidade Federal Fluminense. Rio de Janeiro-RJ-Brasil. ${ }^{2}$ Enfermeira. Doutora em enfermagem. Universidade Federal Fluminense. Rio de Janeiro-RJ-Brasil. ${ }^{3}$ Enfermeiro. Doutor em enfermagem. Universidade Federal Fluminense. Rio de Janeiro-RJ-Brasil. ${ }^{4}$ Enfermeira. Doutora em enfermagem. Universidade Federal do Rio de Janeiro. Rio de Janeiro-RJ-Brasil. ${ }^{5}$ Enfermeira. Mestre em enfermagem. Universidade Federal Fluminense. Rio de Janeiro-RJ-Brasil.

RESUMO: Estudo descritivo, cujo objetivo fora validar o diagnóstico de enfermagem Dor Óssea no mieloma múltiplo e suas intervenções de enfermagem. Realizada busca bibliográfica para construção das afirmativas e utilizada a Classificação Internacional para a Prática de Enfermagem ${ }^{\circledR}$. Em seguida, por meio de formulário, as afirmativas foram submetidas à validação de conteúdo por especialistas em enfermagem oncológica atuantes num hospital universitário. A análise ocorreu por meio de estatística simples, considerando a variável de interesse: índice de concordância, com resultado superior a 0,75. O diagnóstico de Dor Óssea e as intervenções atingiram índice de concordância de 1,0 e de 0,90 respectivamente. A validação aponta para a relevância do diagnóstico de enfermagem nos pacientes com mieloma múltiplo e reflete a possibilidade de melhoria da qualidade assistencial através das intervenções de enfermagem padronizadas.

DESCRITORES: Processos de enfermagem; Diagnóstico de enfermagem; Mieloma múltiplo.

\section{VALIDATION OF THE DIAGNOSIS BONE PAIN AND ITS NURSING INTERVENTIONS IN MULTIPLE MYELOMA}

\begin{abstract}
Descriptive study aimed at validating the nursing diagnosis Bone Pain in multiple myeloma and its nursing interventions. A bibliographic search was undertaken to construct the assertions and the International Classification for Nursing Practice ${ }^{\circledR}$. Next, using a form, the assertions were submitted content validation by cancer nursing specialists active at a university hospital. The analysis was carried out through simple statistics, in view of the variable of interest: agreement level, with a result superior to 0.75 . The diagnosis Bone Pain and the interventions reached an agreement rate of 1.0 and 0.90 , respectively. The validation indicates the relevance of the nursing diagnosis in patients with multiple myeloma and reflects the possibility to improve the quality of care through standardized nursing interventions.
\end{abstract}

DESCRIPTORS: Nursing processes; Nursing diagnosis; Multiple myeloma.

\section{VALIDACIÓN DEL DIAGNÓSTICO DOLOR ÓSEO Y SUS INTERVENCIONES DE ENFERMERÍA EN MIELOMA MÚLTIPLO}

RESUMEN: Estudio descriptivo, cuyo objetivo fue validar el diagnóstico de enfermería Dolor Óseo en el mieloma múltiplo y sus intervenciones de enfermería. Fue realizada búsqueda bibliográfica para construcción de las afirmativas y utilizada la Clasificación Internacional para la Práctica de Enfermería ${ }^{\circledR}$. Después, por medio de formulario, las afirmativas fueron sometidas a la validación de contenido por especialistas en enfermería oncológica actuantes en un hospital universitario. El análisis ocurrió por medio de estadística simple, considerando la variable de interés: índice de concordancia, con resultado superior a 0,75. El diagnóstico de Dolor Óseo y las intervenciones atingieron índice de concordancia de 1,0 y de 0,90 respectivamente. La validación apunta para la relevancia del diagnóstico de enfermería en los pacientes con mieloma múltiplo y reflecte la posibilidad de mejoría de la cualidad asistencial por medio de las intervenciones de enfermería tipificadas. DESCRIPTORES: Procesos de enfermería; Diagnóstico de enfermería; Mieloma múltiplo.

*Artigo extraído da dissertação de mestrado intitulada: Subconjunto de conceitos da Classificação Internacional para a Prática de Enfermagem para o cuidado aos pacientes com mieloma múltiplo. Universidade Federal Fluminense, 2012.

Autor Correspondente:

Silvia Maria de Sá Basílio Lins

Universidade Federal Fluminense

Rua Bento Lisboa nº120 - 22221-011 - Rio de Janeiro-RJ-Brasil.

E-mail: silviamarialins@gmail.com
Recebido: 29/05/2014 Finalizado: 10/10/2014 


\section{INTRODUÇÃO}

O mieloma múltiplo é uma neoplasia maligna originada do crescimento indiscriminado de plasmócitos na medula óssea, provocando sua sobrecarga e suprimindo a produção das demais células, o que ocasiona o acúmulo de imunoglobulinas e seus fragmentos no sangue periférico ${ }^{(1)}$. A dor óssea é uma manifestação clínica frequente, ocorrendo em 50 a $90 \%$ dos casos, e está relacionada à destruição óssea, comumente localizada nas costas e coluna dorsal, confirmando que a doença está em plena atividade e se encontra num estágio clínico mais avançado ${ }^{(1,2)}$.

As alterações esqueléticas repercutem para o paciente sob a forma de dor óssea e possíveis fraturas patológicas o que exige da equipe de enfermagem um olhar especializado. Assim sendo, é importante reconhecer os diagnósticos de enfermagem relacionados ao mieloma múltiplo, bem como as intervenções a serem implementadas junto aos pacientes com vistas a sua qualidade de vida, considerando que o mieloma múltiplo é uma doença ainda sem possibilidade de cura.

A utilização dos diagnósticos e das intervenções de enfermagem na prática clínica pode ser facilitada pelo uso de uma linguagem profissional padronizada para o registro diário das etapas metodológicas do processo de enfermagem. Dentre as possíveis linguagens destaca-se a Classificação Internacional para a Prática de Enfermagem - CIPE $®$, que se caracteriza como uma terminologia combinatória que favorece o mapeamento cruzado entre os termos utilizados na prática de enfermagem e as existentes classificações. É um instrumento representativo que descreve a prática de enfermagem por meio de uma combinação de termos descritos em seus sete eixos fundamentais (Foco, Julgamento, Cliente, Ação, Meios, Localização e Tempo) para descrição de diagnósticos, intervenções e resultados de enfermagem ${ }^{(3,4)}$.

Além disso, a utilização da CIPE $®$ como linguagem padronizada para o registro do processo de enfermagem vem ao encontro da Resolução 358/2009 do Conselho Federal de Enfermagem, que regulamenta a implantação da Sistematização da Assistência de Enfermagem - SAE nos serviços de saúde e considera esta como um processo de trabalho adequado às necessidades de saúde da comunidade.
No Brasil, alguns estudos têm sido desenvolvidos com o objetivo de criar subconjuntos de conceitos e/ou validar diagnósticos e intervenções usando a CIPE ${ }^{\circledR(5-7)}$. Esse estudo é um subprojeto da pesquisa Subconjuntos de Conceitos $\mathrm{CIPE}^{\circledR}$ para Pacientes com Mieloma Múltiplo, que tem sido desenvolvido na enfermaria de hematologia de um hospital universitário, na cidade de Niterói.

A elaboração de Subconjuntos de Conceitos da CIPE $^{\circledR}$ tem sido uma estratégia do Conselho Internacional de Enfermeiros - CIE para facilitar o uso dessa classificação durante a execução e registro do Processo de Enfermagem. Os Subconjuntos de Conceitos da CIPE ${ }^{\circledR}$ são compostos por enunciados preestabelecidos de diagnósticos, intervenções e resultados de enfermagem, que podem estar direcionados tanto a clientelas (indivíduo, família e comunidade), quanto a prioridades ou a condições específicas de saúde, ambientes ou especialidades de cuidado, bem como a fenômenos específicos de enfermagem ${ }^{(8)}$.

Dentro do projeto mais amplo de elaboração do Subconjunto CIPE $®$, o diagnóstico de enfermagem Dor Óssea surgiu com bastante freqüência a partir da revisão de literatura, de modo que este estudo apresentou como objetivo validar o diagnóstico de enfermagem Dor Óssea e suas respectivas intervenções de enfermagem. Destaca-se que a validação é uma etapa recomendada pelo CIE para construção dos subconjuntos de conceitos conferindo ao dado validado uma certificação de aplicabilidade na prática clínica ${ }^{(8)}$.

\section{MÉTODO}

Trata-se de estudo exploratório-descritivo, desenvolvido em um hospital universitário, localizado na cidade de Niterói, no Estado do Rio de Janeiro, no ano de 2012. Os participantes receberam um formulário contendo a afirmativa diagnóstica Dor Óssea, com sua definição, e suas respectivas Intervenções de Enfermagem, e deveriam atribuir suas notas à afirmativa diagnóstica e às intervenções propostas.

Os participantes desse estudo, que atuaram na etapa de validação, foram nove enfermeiros atuantes na enfermaria de hematologia e no ambulatório de quimioterapia do cenário em questão, que possuíam cinco anos ou mais de experiência naqueles setores específicos e pós graduação na 
área de oncologia ou hematologia, critérios de inclusão utilizado para seleção dos participantes bem como para considerá-los especialistas na área.

Para alcance do objetivo proposto, o projeto foi executado em duas etapas. A primeira etapa da pesquisa consistiu na formulação da afirmativa diagnóstica Dor Óssea e suas respectivas intervenções, o que ocorreu a partir de um levantamento bibliográfico de artigos nacionais e internacionais na base Literatura científica e técnica da América Latina e do Caribe -LILACS, cujo descritor utilizado foi, unicamente, Mieloma Múltiplo. Foram utilizados como critérios de inclusão para apreciação dos artigos um recorte temporal de cinco anos (2008-2012), idiomas inglês, espanhol e português e disponibilidade dos mesmos na íntegra.

Foram selecionados os artigos que traziam como evidência clínica do mieloma múltiplo a dor óssea, que, em seguida, foi submetida a um mapeamento cruzado com os termos constantes no eixo foco da CIPE $^{\circledR}$ versão 2, tendo sido formulada, portanto, a afirmativa diagnóstica Dor Óssea. A construção das Intervenções de Enfermagem foi baseada em literatura de enfermagem sobre a temática da dor, tanto geral quanto oncológica, e para formulação das afirmativas foram utilizadas as regras estabelecidas pela CIPE ${ }^{\circledR}$, ou seja, utilizou-se um termo do eixo ação e um termo alvo.

Na segunda etapa da pesquisa, a afirmativa diagnóstica de Dor óssea e respectivas Intervenções de Enfermagem foram submetidas ao processo de validação de conteúdo por especialistas, os nove enfermeiros participantes do estudo. Para a validação, foi considerada a variável de interesse Índice de Concordância. O processo de validação (quadro 1) consistiu em atribuir uma nota que variava de 1 a 5: Nota 1 - não se aplica; Nota 2 - se aplica muito pouco; Nota 3 - se aplica de algum modo; Nota 4 - se aplica consideravelmente; Nota 5 - é muito característica. Cada nota recebeu um peso: 1 $=0 ; 2=0,25 ; 3=0,50 ; 4=0,75$ e $5=1$. Foi então realizada a média ponderada dos valores atribuídos por todos os participantes da pesquisa, e foram consideradas válidas a afirmativa de diagnóstico e suas respectivas intervenções com Índice de Concordância superior a 0,75.

Este projeto foi aprovado pelo Comitê de Ética em Pesquisa da instituição onde foi desenvolvido, com número de registro 369/11 de 02/12/2011.

Quadro 1 - Processo de validação do diagnóstico de enfermagem dor óssea e de suas intervenções de enfermagem. Niterói-RJ-Brasil, 2013.

\begin{tabular}{|l|l|l|}
\hline Nota atribuída & Significado & Peso \\
\hline 1 & Não se aplica & 0 \\
\hline 2 & Se aplica muito pouco & 0,25 \\
\hline 3 & Se aplica de algum modo & 0,50 \\
\hline 4 & Se aplica consideravelmente & 0,75 \\
\hline 5 & É muito característica. & 1 \\
\hline
\end{tabular}

\section{RESULTADOS}

Do levantamento na base de dados, emergiram 30 artigos referentes à temática do mieloma múltiplo, dos quais 15 (50\%) artigos traziam a dor óssea como uma evidência clínica da patologia. Realizando o mapeamento cruzado desta com os termos do

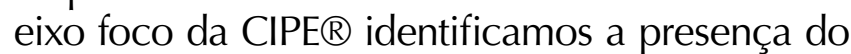
termo dor óssea na classificação, a partir da qual elaboramos a afirmativa diagnóstica Dor Óssea.

Em seguida, partindo da literatura nacional e internacional de enfermagem, que abrangia tanto a dor geral quanto a dor oncológica, foram construídas as intervenções de enfermagem específicas para o diagnóstico elaborado.
Também foram seguidas as orientações da CIPE $\mathbb{R}$ para construção das mesmas. As intervenções de enfermagem estão apresentadas no Quadro 2.

Quando apresentado aos especialistas, o diagnóstico de Dor Óssea atingiu um índice de concordância de 1, o que significa que todos os avaliadores atribuíram a este diagnóstico o grau máximo de concordância. Quanto às Intervenções de enfermagem, a avaliação das mesmas se deu em conjunto, ou seja, todas as intervenções de enfermagem agrupadas receberam uma nota de cada especialista. $\mathrm{O}$ índice de concordância para este conjunto de intervenções foi de 0,90 , não tendo havido sugestão de inclusão de outras intervenções de enfermagem para o diagnóstico em questão. 
Quadro 2 - Diagnóstico de enfermagem dor óssea e suas respectivas intervenções de enfermagem no mieloma múltiplo. Niterói-RJ-Brasil, 2013

\begin{tabular}{|c|c|}
\hline Diagnóstico de enfermagem & Intervenções de enfermagem \\
\hline $\begin{array}{l}\text { DOR ÓSSEA: dor musculoesquelética com sensação } \\
\text { que é originada no periósteo, fratura comprimida; as } \\
\text { sensações são normalmente descritas como dores pro- } \\
\text { fundas, que são penetrantes e estão presentes durante o } \\
\text { período de repouso e quando imóvel }{ }^{(7)} \text {. }\end{array}$ & $\begin{array}{l}\text { Estabelecer distinção entre a dor e o sofrimento. } \\
\text { Identificar atitude sobre a dor. } \\
\text { Ensinar o gerenciamento da dor. } \\
\text { Minimizar o medo do paciente } \\
\text { Orientar quanto ao regime medicamentoso e efeito } \\
\text { adverso. } \\
\text { Manter a dignidade e a privacidade do paciente quanto } \\
\text { a sua dor. } \\
\text { Avaliar se a dor é branda, moderada ou severa. } \\
\text { Iniciar analgesia controlada pelo paciente. } \\
\text { Promover alívio da dor com analgésico prescrito. } \\
\text { Administrar analgésico antes da movimentação corpo- } \\
\text { ral. } \\
\text { Avaliar resposta à terapia para dor. } \\
\text { Gerenciar resposta negativa ao tratamento. } \\
\text { Explicar e promover as medidas não invasivas e não } \\
\text { farmacológicas de alívio da dor: posicionamento } \\
\text { apropriado, técnica de distração (musicoterapia, con- } \\
\text { versa, televisão, entre outros), exercícios respiratórios, } \\
\text { massagens, aplicação de aparelho de aquecimento/ } \\
\text { resfriamento e técnicas de relaxamento. } \\
\text { Consultar para gerenciamento da dor. } \\
\text { Demonstrar aceitação da dor do paciente. } \\
\text { Encorajar caminhar sem auxílio, se possível. } \\
\text { Encorajar afirmações positivas. }\end{array}$ \\
\hline
\end{tabular}

\section{DISCUSSÃO}

O mieloma múltiplo já foi classificado como câncer ósseo, sendo mais tarde incluído entre as doenças oncohematológicas ${ }^{(9)}$. A dor óssea é um dos componentes da tríade que caracteriza a doença e uma das complicações mais comuns do mieloma, estando relacionada à destruição do osso $^{(10-11)}$, a qual resulta de um desequilíbrio na formação e reabsorção óssea ${ }^{(11)}$. Ocorre devido ao aumento da atividade osteoclástica provocada pelos fatores ativadores produzidos pelas células mielomatosas, levando a intensa reabsorção óssea, com perda óssea disseminada, lesões líticas e fraturas ${ }^{(10,13)}$.

As dores intensas interferem significativamente sobre a pessoa, prejudicando seu desempenho cotidiano e sua qualidade de vida. No mieloma, pode ser descrita como intolerável, podendo ainda ser refratária ao tratamento medicamentoso mais comum (com não opióides ou com opióides fracos). Daí a importância das intervenções de enfermagem, considerando que podem proporcionar apoio emocional e segurança, encorajando o cliente a compartilhar suas dúvidas, reduzindo medos e ansiedades, bem como, gerando condições satisfatórias ao enfrentamento da dor, além de propiciar oportunidades para que o enfermeiro forneça retroalimentação realista e tranquilização ${ }^{(14-16)}$.

Quando os enfermeiros validam as intervenções de enfermagem propostas, consideram que tais atividades são realizadas cotidianamente junto aos pacientes com Mieloma Múltiplo. Estas intervenções são justificadas na literatura como: Consultar para gerenciamento da dor, realizada a fim de se obter um controle apropriado da dor; Demonstrar aceitação da dor do paciente, deve-se transmitir ao paciente a impressão de que sua dor é compreendida e que ela pode ser controlada; Estabelecer distinção entre a dor e o sofrimento, o conceito de dor envolve os componentes sensitivo-discriminativo (sensação física), afetivomotivacional (emoções) e cognitivo-avaliativo (pensamento) $^{(17)}$. A dor resulta da interação do aspecto físico e químico, do estímulo nocivo e da sua interação com fatores emocionais e culturais do indivíduo relacionados com dor, constitui-se, portanto, em uma experiência privada e subjetiva, não sendo resultado somente de características de lesão tecidual ${ }^{(18)}$. 
Identificar atitude sobre a dor, os sinais visíveis de dor (físicos e comportamentais) são determinados pela tolerância do indivíduo a ela e pela sua duração, não por sua intensidade, além disso, os fatores culturais da situação específica interferem em como o indivíduo expressa a dor ${ }^{(18,19)}$; Ensinar o gerenciamento da dor, já que o controle eficiente da dor pode melhorar, consideravelmente, o funcionamento físico geral ${ }^{(19)}$; Minimizar o medo do paciente, pessoas preparadas para procedimentos dolorosos por meio de explicações das verdadeiras sensações apresentam menos estresse do que as que recebem explicações vagas ${ }^{(19)}$.

Orientar quanto ao regime medicamentoso e efeito adverso. A orientação em relação ao manejo dos efeitos adversos deve seguir o protocolo elaborado pela equipe, com base na literatura científica e na experiência clínica dos membros constituintes da equipe; Manter a dignidade e a privacidade do paciente quanto a sua dor; proporcionando um ambiente terapêutico em relação à temperatura, umidade, ventilação, luminosidade e ruídos ${ }^{(19)}$; Avaliar se a dor é branda, moderada ou severa utilizando a escala de intensidade de dor mais apropriada ${ }^{(14,19)}$.

Iniciar analgesia controlada pelo paciente, evita os retardos inerentes à administração do analgésico; Promover alívio da dor com analgésico prescrito, a via de administração oral deve ser a escolhida quando possível, quando for para administrações frequentes, utilizar via venosa, uma vez que a absorção é garantida e não dolorosa ${ }^{(19)}$; Administrar analgésico antes da movimentação corporal , a abordagem preventiva pode reduzir a dose total diária e diminuir a ansiedade de solicitar a medicação e esperar seu efeito; Avaliar resposta à terapia para dor solicitando ao paciente que avalie a intensidade da dor antes da medicação e o tipo de alívio obtido com a mesma meia hora após sua administração ${ }^{(18)}$; Gerenciar resposta negativa ao tratamento, reavaliando a dor com frequência e em caso de resposta negativa, modificar o plano de cuidado $^{(15)}$.

Explicar e promover as medidas não invasivas e não farmacológicas de alívio da dor: posicionamento apropriado, técnica de distração (musicoterapia, conversa, televisão), fazer exercícios respiratórios, aplicar aparelho de aquecimento/resfriamento e técnicas de relaxamento, tais medidas podem incrementar os efeitos terapêuticos dos analgésicos e proporcionar uma crescente sensação de controle e de envolvimento ativo. Além disso, reduz o estresse, diminui a fadiga física, abaixa o limiar dos estímulos sensoriais, induz a visualização e facilita o fluxo de energia corporal por meio do reflexo talâmico ${ }^{(17,18)}$.

Encorajar caminhar sem auxílio, se possível, promovendo a mobilidade ideal e discutindo o valor dos exercícios para o fortalecimento e o alongamento dos músculos; Encorajar afirmações positivas, elogiando o paciente pela sua resistência e convencendo-o do controle da dor, independente da forma como ele se comportou. Isto ajuda o paciente a transformar os comportamentos gerados pela dor em comportamentos saudáveis e de bem estar ${ }^{(15)}$.

As intervenções de enfermagem ora propostas visam o alívio da dor derivada do câncer. São intervenções que buscam criar uma relação de confiança profissional-paciente, controlar as condições ambientais e proporcionar uma sensação de conforto para com isso facilitar o controle da dor.

\section{CONCLUSÃO}

O diagnóstico de enfermagem Dor Óssea atingiu o índice máximo de concordância entre os especialistas desta pesquisa. Alto índice também foi apresentado pelo conjunto das intervenções de enfermagem propostas. Destaca-se a versatilidade da CIPE $^{\circledR}$, que atende a uma multiplicidade de repostas humanas, adaptando-se, facilmente, à cultura de trabalho local; considerando que as afirmativas propostas foram bem aceitas e bem compreendidas pelos participantes do estudo.

Esta pesquisa, como parte de um projeto maior de construção de Subconjunto de conceitos de diagnósticos e intervenções de enfermagem para o mieloma múltiplo, vem colaborar com os esforços institucionais do local de desenvolvimento da pesquisa para formalização e registro do processo de enfermagem no setor de hematologia e quimioterapia. O processo de validação, pelos enfermeiros envolvidos no cuidado, permitiu a construção de afirmativas comumente utilizadas nesta área específica e que devem servir de referência para estes profissionais. 
Apresenta-se um passo importante para o uso de um sistema de classificação na prática clínica da enfermagem que permite a padronização da linguagem, a mensuração dos resultados alcançados nas ações de cuidado, bem como subsídios para uma prática baseada em evidências, o que contribui para a melhoria da qualidade assistencial.

\section{REFERÊNCIAS}

1. Lorenzi TF. Manual de hematologia: propedêutica e clínica. 4a ed. Rio de Janeiro: Guanabara Koogan; 2011.

2. Silva ROP, Branda KMA, Pinto PVM, Faria RMD, Clementino NCD, Silva CMS, et al. Mieloma múltiplo: características clínicas e laboratoriais ao diagnóstico e estudo prognóstico. Rev Bras Hematol Hemoter. [Internet] 2009; 31(2). [acesso em 12 jun 2013]. Disponível: http://www.scielo.br/scielo. php?pid=S1516-84842009000200005\&script $=$ sci arttext. doi: http://dx.doi.org/10.1590/S151684842009005000013

3. Lins SMSB, Santo FHE, Fuly PSC, Garcia TR. Subconjunto de conceitos diagnósticos da CIPE $®$ para portadores de doença renal crônica. Rev. bras. enferm. 2013; 66(2): 180-9.

4. Santana LL, Mazza VA, Taube SAM, Sarquis LMM. Diagnósticos e intervenções de enfermagem em unidade de pronto atendimento à luz das necessidades humanas básicas. Cogitare Enferm. 2011; 16(4):675-81.

5. Carvalho MWA, Nóbrega MML, Garcia TR. Processo e resultados do desenvolvimento de um Catálogo

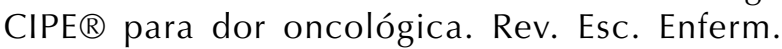
USP. [Internet] 2013; 47(5) [acesso em $01 \mathrm{fev}$ 2014]. Disponível: http://www.scielo.br/scielo. php?pid=S0080-62342013000501060\&script=sci arttext\&tlng=pt. doi: http://dx.doi.org/10.1590/S0080623420130000500008

6. Medeiros ANT, Nóbrega MML. Terminological subsets of the international classification for nursing practice - ICNP® for senior patients: a methodological study. Online Braz J Nurs. [Internet] 2012, 11 (2). [acesso em 01 fev 2014]. Disponível: http://www.objnursing.uff.br/ index.php/nursing/article/view/4013. doi: http://dx.doi. org/10.5935/1676-4285.2012S007

7. Fialho LFG, Fuly PSC. CIPE® catalogue for patients with multiple myeloma: a descriptive study. Online Braz J Nurs. [Internet] 2012; 11(2) [acesso em 15 mar 2013]. Disponível em http://www.objnursing.uff.br/ index.php/nursing/article/view/4013. doi: http://dx.doi. org/10.1590/S1516-84842009005000013 internacional para a prática de enfermagem versão 2 . São Paulo: Algol; 2011.

9. Sakae TM, Santos NAF, Baldessar MZ. Sobrevida de pacientes portadores de mieloma múltiplo atendidos em hospital de referência no sul de Santa Catarina. Rev Bras Clin Med. 2010; 8(3): 216-21

10. Andrade VP. Aspectos morfológicos da infiltração da medula óssea por condições, exibindo diferenciação plasmocítica e gamopatia monoclonal. Rev Bras Hematol Hemoter. 2009; 3 (4): 273-9.

11. Santos PSS, Gambirazi LM, Felix VB, Magalhães MHCG. Osteonecrose maxilar em pacientes portadores de doenças neoplásicas sob o uso de bisfosfonatos. Rev Bras Hematol Hemoter. 2008; 30(6): 501-4.

12. Heinem JR; Santos JS. Mieloma múltiplo com fratura no colo do úmero: relato de caso. Rev. HCPA 2010; 30(1): 68-72.

13. Leão $E R$, Chaves LD. Dor: $5^{\circ}$ sinal vital: reflexões de enfermagem. $2^{a}$ ed. São Paulo: Martinari; 2007.

14. Doenges ME, Moorhouse MF, Murr AC. Diagnóstico de enfermagem: intervenções, prioridade, fundamentos. Rio de Janeiro: Guanabara Koogan; 2012.

15. Santos AG. Characterization of cancer pain in patients submitted to radiotherapy. Rev enferm UFPE. 2012; 6(9): 2111-8.

16. Pimenta CAM, Santos EMM, Chaves LD, Martins LM, Gutierrez BAO. Controle da dor no pós-operatório. Rev. Esc. Enferm. USP. 2001; 35 (2): 180-3.

17. Budó MLD, Nicolini D, Resta DG, Büttenbender, Pippi MC e Ressel LB. A cultura permeando os sentimentos e reações frente a dor. Rev. Esc. Enf. USP. 2007; 41(1): 36-46.

18. Barbosa MH, Araújo NF, Silva JAJ, Corrêa TB, Moreira TM, Andrade EV. Avaliação da intensidade da dor e analgesia em pacientes no período pós-operatório de cirurgias ortopédicas. Esc. Anna Nery. 2014; 18(1):143-7.

19. Carpenito LJ. Diagnósticos de enfermagem: aplicação à prática clínica. 8ª Ed. Porto Alegre: Artmed; 2002.

8. Conselho Internacional de Enfermeiros. Classificação 